\title{
Diagnostic and Treatment Challenges in Acute Myocardial Infarction: Perspectives from a Community Hospital
}

François M.A. Paris ${ }^{1 *}$, Gillian J. Jessurun ${ }^{2}$, Rutger L. Anthonio ${ }^{2}$, Massimo A. Mariani ${ }^{3}$

'House officer cardiology, Department of Cardiology, Treant Zorggroep Scheper Ziekenhuis Emmen, The Netherlands ${ }^{2}$ Intervention cardiologist, Department of Cardiology, Treant Zorggroep Scheper Ziekenhuis Emmen, The Netherlands ${ }^{3}$ Cardiothoracic surgeon Heart, Center University Medical Center Groningen, The Netherlands

\section{Article Info}

\section{Article Notes}

Received: August 16, 2020

Accepted: October 14, 2020

\section{*Correspondence:}

Mr. F.M.A. Paris, Treant Zorggroep Loc Scheper Emmen,

Boermarkeweg 60, 7824 AA Emmen, The Netherlands; Telephone No: +31 683167580; Email: f.paris@treant.nl.

(c) 2020 Paris FMA. This article is distributed under the terms of the Creative Commons Attribution 4.0 International License.

\section{Keywords:}

STEMI

Acute coronary syndrome

Primary PCl

Treatment options

\section{Abstract}

One of the most important causes of death in Western society, following cancer, is myocardial infarction (MI). Although acute $\mathrm{Ml}$ occurs at an older age, the incidence of acute $\mathrm{MI}$ in younger adults has increased.

Essentially, treatment of acute $\mathrm{MI}$ is reperfusion therapy, which should preferably be performed within 12 hours of onset of the symptoms. Time is imperative and shorter intervals between symptoms an reperfusion leads to lower mortality. Conversely, a longer interval results in higher mortality, more mechanical complications and morbidity.

At our institution, located in the middle of a thinly populated rural area, patients used to be treated with thrombolysis until 2005. The nearest centers for percutaneous coronary interventions ( $\mathrm{PCI}$ ) and cardiothoracic surgery, had a travelling distance of 60 minutes. Since the introduction of interventional cardiology in the province of Drenthe, we managed to reduce the symptom to needle time.

Recently, we have been confronted with the COVID-19 pandemic, led to an increase in patient's and doctor's delay. This unfolded new challenges in treating acute $\mathrm{MI}$.

This overview addresses the general clinical approach of acute $\mathrm{MI}$ and highlights the diagnostic approach and treatment options of both premature atherosclerosis and non-atherosclerotic causes of MI from our clinical perspective. We would like to argue that a personalized clinical approach remains of utmost importance in each patient treated by protocolized medicine.

\section{Introduction}

Myocardial infarction (MI) is the most important cause of death globally, which costs almost 18 million lives annually. These deaths occur mainly in patients above the age of 70 years. A third occurs in patients under 70 years of age and in young patients the incidence fortunately is rare $(<10 \% \text { of patients is younger than } 40 \text { years })^{1}$. Due to risk factors as smoking and hypertension, this latter group has been steadily growing, however. Since an acute MI is not considered as the most likely cause of chest pain in young adults, we argue that the risk of underdiagnosis and/or delayed treatment may be significant in this category of patients.

Up until 10 years ago, interventional cardiology was not available in our region in the province of Drenthe in the Netherlands. Patients used to be treated with fibrinolysis and subsequently a small portion would be transferred to other clinics where reperfusion therapy would be performed, days or maybe even weeks after the first admission. 
Since we started to provide invasive cardiac care at our institution, we have been part of the regional network of acute cardiac care, consisting of general cardiologists, interventional cardiologists, general physicians and the medical director of the regional ambulance service. In this meeting, agreements are made to optimize service delivery. Due to this network, patients were referred much earlier to our hospital, the interventional cardiologist would be supplied with relevant information beforehand and coronary angiographies could be performed much earlier than before. The primary goal was to restore antegrade reperfusion as soon as possible. In the second phase the goal was, to obtain sustainable beneficial effect of reperfusion in these patients.

During the COVID-19 pandemic, we have seen delays in patient presentation due to their fear of getting infected in the hospital or the hesitation to be a burden to the hospital which presumably was overwhelmed by the care for COVID-19 patients. Another issue was that patients as well as general physicians interpreted atypical symptoms as being caused by the COVID-19 virus, thus causing a twoweek delay due to quarantining.

\section{A Dutch Rural Perspective}

We would like to highlight that this review addresses the clinical approach of patients with an acute myocardial infarction in general, from the perspective of a PCI center located within a rural area. In our setting the nearest Clinic with cardiothoracic surgery is a 45-minute drive away.

The clinical approach of acute MI remains challenging as the diagnosis and treatment requires meticulous reasoning to treat the culprit lesion but at the same time extends beyond the culprit lesion towards the complete patient. When reviewing the ECG, it is important that the phase of ischemia is qualified: hyperacute ischemia, fully evolved phase or chronic stabilized phase. At first, there is a hyperacute phase with large symmetrical or peaked T-waves. These are followed by convex ST-segment elevation, which represents a completely developed ischemic phase. Finally, a chronically stabilized phase develops, in which negative $\mathrm{T}$ waves are replaced by positive T waves.

The presence of multiple risk factors, but also age and sex, facilitate the clinical conception of vessel abnormalities that can be found, thus enabling a better planning of an adequate treatment strategy.

In older age groups, the main cause of acute MI is atherosclerotic disease. When treating a younger patient with chest pains other pathophysiologic mechanisms should be considered as well. In younger patients underlying causes are premature atherosclerosis or nonatherosclerotic factors ${ }^{1-3}$.
1) Premature atherosclerosis - development of coronary atherosclerosis at a young age is correlated with the presence of conventional cardiovascular risk factors such as smoking, hypertension, dyslipidemia, overweight, inactivity and stress ${ }^{1}$. Smoking is one of the most impo rtant risk factors. Frequent exposure to cigarette smoke stimulates release of catecholamines, which causes damage to the endothelial cells. This can lead to vascular intima dysfunction already at a young age. This process involves alternating low level cholesterol deposition and platelet aggregation (plaque formation). The lipid core within this plaque formation may rupture. This results in release of vasoactive factors leading to acute vascular occlusion. Genetic predisposition also increased the risk of premature atherosclerosis, such as in the case of a mutation in the factor V-Leiden gene or genetic hypercholesterolemia ${ }^{4}$.

Treatment of acute MI due to premature atherosclerosis consists of protocollary revascularization by means of primary PCI, followed by cardiovascular risk management of risk factors.

2) Non-atherosclerotic cause - In this condition the mechanism of coronary occlusion differs ${ }^{1,2}$. This may result from various underlying disorders that are accompanied by unconventional risk factors in some cases, such as pregnancy and direct contact sports. In table 1, an overview of non-atherosclerotic causes is depicted. For example, the occlusion can be induced by coronary spasms, embolization through coronary arteries due to endocarditis or secondary thrombus formation. The development of secondary thrombosis could be explained by the Virchow triad (figure 1$)^{5}$. This theory describes that development of thrombosis that can be triggered by three factors: stasis of blood flow, endothelial damage and hypercoagulability or abnormal blood composition.

Coronary spasms can also cause an increased risk of secondary thrombosis because these spasms induce minor damage to the vascular endothelium and activate coagulation. Both cocaine and binge drinking can cause coronary spasms $\mathrm{s}^{1,6,7}$.

Table 1: Overview of mechanisms of a MI with a non-atherosclerotic cause.

\begin{tabular}{|l|l|} 
Mechanism & Explanation \\
\hline Spasm coronary artery & drug- and excessive alcohol use \\
\hline $\begin{array}{l}\text { Aberrant anatomy of the coronary } \\
\text { arteries }\end{array}$ & aberrant course between the aorta and pulmonary artery \\
\hline Systemic inflammatory disease & $\begin{array}{l}\text { systemic lupus erythematodes, rheumatoid arthritis, Wegener } \\
\text { granulomatosis }\end{array}$ \\
\hline Coagulation disorder & polycythaemia vera, thalassemia \\
\hline Coronary mimicry & $\begin{array}{l}\text { cerebro-vascular accident, pneumothorax, pulmonary } \\
\text { embolus, pericarditis, pancreatitis, pyelonefritis, } \\
\text { hyperkaliemia, metabolic acidosis }\end{array}$ \\
\hline Pregnancy & $\begin{array}{l}\text { change of hemodynamic state (increase in the amount of } \\
\text { blood and coagulants), mucoid degeneration }\end{array}$ \\
\hline Endothelial damage & $\begin{array}{l}\text { blunt trauma on the chest, spontaneous dissection of the } \\
\text { coronary artery, potentially also in case of deceleration } \\
\text { trauma }\end{array}$ \\
\hline
\end{tabular}




\section{Virchow's Triad}

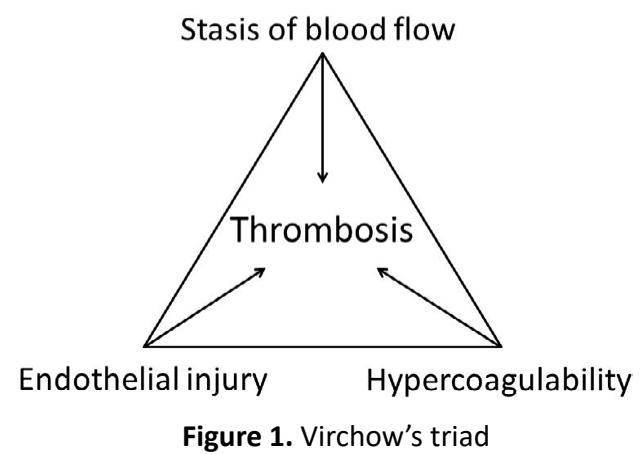

An occlusion of the coronary arteries could also be caused by a damaged vascular endothelium, coronary spasms or a rupture of an existing plaque after blunt trauma to the chest ${ }^{8}$. This may occur during direct trauma, ball sports, cycling sports and also motorsports. The damage to the vascular endothelium may induce locally increased coagulation with formation of thrombosis.

During intense exercise or sporting activities there are other mechanisms which may cause an acute MI. A relative dehydration due to strenuous exercise could increase the viscosity of the blood, which in turn could also contribute to an acute thrombus formation'. Deceleration trauma, as occurs in off-road (motor or cycling) sports may result in acute MI, due to vascular ruptures, vascular spasms, embolism to the coronary arteries or displacement of an atherosclerotic plaque ${ }^{17}$.

In young women, acute MI is rare because of the protective effect of estrogens ${ }^{10}$. However, there are a few exceptions. First, the risk of developing acute MI is increased during and after pregnancy in the postpartum period (up to 356 days after delivery). During pregnancy, there is an increase in blood volume of 140 to $150 \%$ and increased levels of factor VIII and von Willebrand factor. These are essential coagulation proteins. Simultaneously, levels of protein $S$ are decreased (a vitamin K-dependent anticoagulant). The change in blood composition decreases the risk of bleeding during childbirth, but increases the risk of developing thrombosis and subsequently the risk of acute $\mathrm{MI}^{11}$. Also, evidence has found that mucoid degeneration of the intima in the coronary arteries due to pregnancy could lead to coronary ischemia ${ }^{12}$. Use of contraceptive pills combined with smoking increases the risk of coronary ischemia in women. Additionally, spontaneous dissection of the coronary arteries is more prevalent in women compared to men $^{13,14}$.

In cases of acute MI with a non-atherosclerotic cause, treatment should be individualized with regard to the underlying mechanism. For example, coronary vascular spasm should be treated with non-specific vasodilators (e.g. nitrates) $)^{15}$. Adequate lifestyle advices should be provided, like discouragement of the use of cocaine and drinking large amounts of alcohol.

After establishing the diagnosis and the indication for revascularization therapy, a primary $\mathrm{PCI}$ is performed as soon as possible. As per protocol of the European Society of Cardiology (ESC) we performed a PCI of the culprit coronary artery. When encountering additional lesions, we usually perform a staged second PCI within days to weeks of the primary PCI. It was remarkable to discover that the population of Drenthe suffer from diffuse coronary artery disease. Even at a relatively young age three vessel disease is often encountered in patients presenting with a STEMI or NSTEMI, which makes identifying the culprit a challenge. We have daily heart team conferences with cardiothoracic surgeons of two larger hospitals to determine the best strategy for these patients. When surgical revascularization is indicated, our patients can usually be transferred to these hospitals within a week, to undergo treatment.

Alternative Treatment Options in Acute MI. Restoration of Flow Through the Culprit, What's Next?

We would like to present two cases in which we highlight different strategies to be considered to achieve myocardial flow restoration in acute MI.

\section{Deferred stenting vs direct stenting}

CASE 1 - A 67 year old healthy male was admitted to the hospital because of ongoing chest pains which had begun in the early morning hours. There was a history of short periods of chest pain that disappeared spontaneously within minutes after their onset. The first responders obtained an ECG which showed ST-elevations in the anterolateral leads.

Uponarrivalin thehospital thepatientwashemodynamically stable. The ECG showed a sinus rhythm with a Right Bundle Branch Block (RBBB) and marked ST elevations in V2 - V6 and in a lesser extent also in I and aVL (Figure 2).

Coronary angiography showed an occlusion in the proximal LAD with a high thrombotic load. Therefore, thrombus aspiration was performed after successful recanalization. Hereafter, TIMI III flow was observed. After stenting however, despite a high dosage of intracoronary adenosine and nitroglycerine, flow deterioration was seen. This was due to the deployment of a large stent in a high thrombotic state, with more micro-embolization and subsequent deterioration of TIMI flow. A strategic therapeutic option was to defer stenting for several days.

Occasionally high thrombotic load in the vessel accompanied by low systemic blood pressure can easily result in slow or no reflow when the vessel is stented. This 




Figure 2. Sinus rhythm, RBBB, ST elevation V2 - V6 and in I, aVL

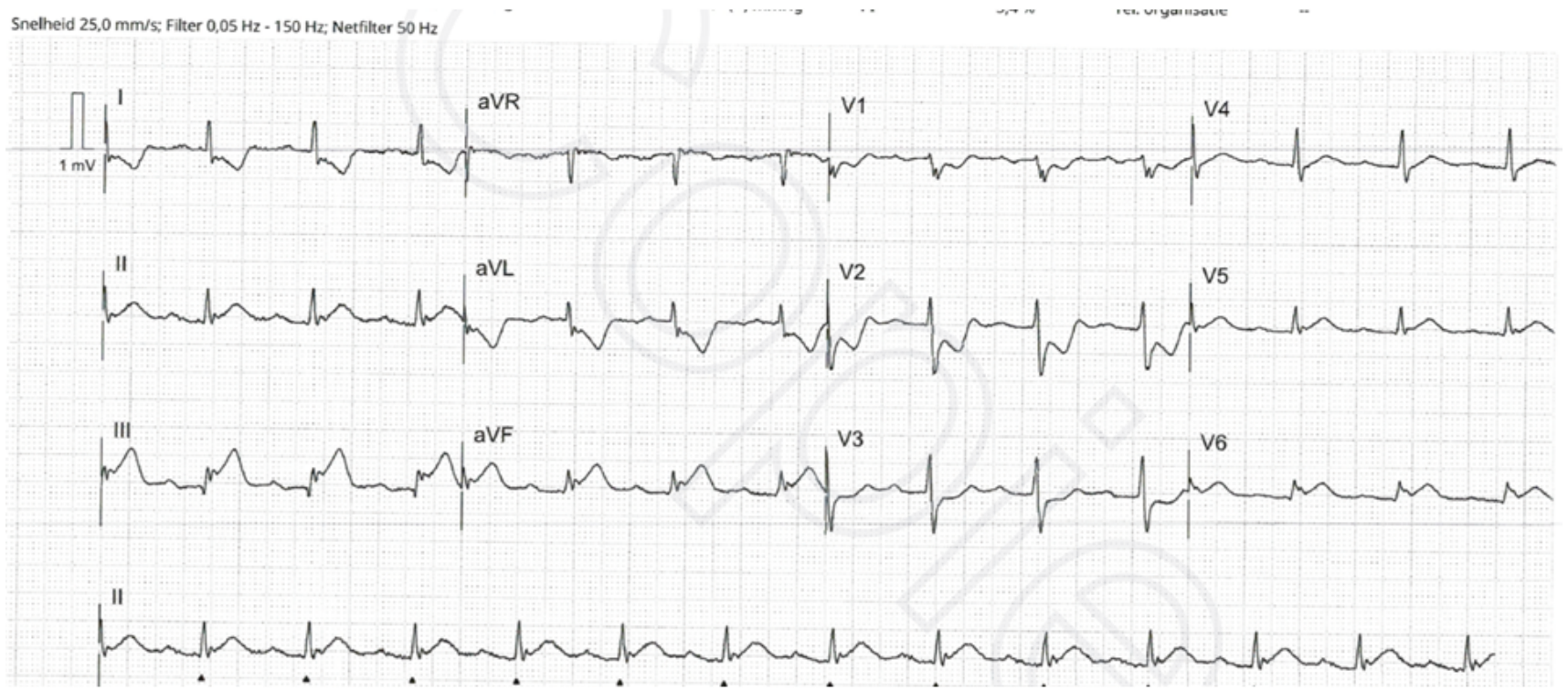

Figure 3. Sinus rhythm with ST-elevations in II, III, aVF, V5 and V6 with marked ST-depressions in V1, V2 as well as in I and aVL

mechanism is known as distal embolization and secondary spasm in the microcirculation of the myocardium. Therefore, it may be conceptualized that deferred stenting may be an exit strategy to reduce the occurrence of slow flow and no reflow following initial restoration of TIMI III flow in the occluded vessel ${ }^{16}$.

\section{AMI during COVID-19 outbreak}

CASE 2 - An 81 year old female patient with a history of coronary heart disease with previous revascularization of the RCA, paroxysmal atrial fibrillation and a non-Hodgkin Lymphoma, was admitted to the hospital with chest pain, ongoing for two days. On admission her ECG showed sinus rhythm with ST-elevations in II, III, aVF, V5 and V6 with marked ST-depressions in V1, V2 as well as in I and aVL, compatible with an infero-postero-lateral STEMI (Figure 3). Since the symptoms had been persisting for a period longer than 12 hours, there was a risk of underlying 
mechanical complication. We evaluated the patient with pre-procedure echocardiography, which showed a thin layer of pericardial effusion, but no signs of any mechanical complications. Subsequent coronary angiography showed an $80 \%$ stenotic lesion in the proximal LAD, as well as a total occlusion of the CX and a total occlusion of the RCA mid segment just proximal of the old stents. A primary PCI of the RCA as the culprit vessel was performed using a $2.75 \times 15 \mathrm{~mm}$ Drug Eluting Stent, followed by a proximal optimization by means of a 3.5 balloon. An attempt was made to revascularize the $\mathrm{CX}$, but it appeared to be a total chronic occlusion.

A few days later an additional PCI of the LAD was performed.

To determine the viability of the lateral wall, a cardiac MRI was made. This showed a transmural basal and inferolateral infarction without viability. Therefore, the decision was made to refrain from treating the CTO of the circumflex.

Late STEMI presentation in the North eastern part of the Netherlands is a relatively frequent observation mostly due to the patient's delay. This may result in a clinical presentation after 1-3 days following initial complaints. First medical contact is subsequently sought at the time the patient experiences complaints of equal severity for 3 days earlier. This warrants a mechanical complication. Before immediate coronary angiography is performed, we recommend investigate the patient for a possible murmur and then perform quick bedside echocardiography to detect a mechanical complication in suspected situations. General awareness to seek medical aid following acute pain in the chest with nausea and vomitus is absent. This cultural dependent personal conviction is worsened during COVID-19 outbreak. The general population in this region is afraid to be infected at the hospital and have the feeling that they disturb the already busy physicians and other medical personnel during the outbreak.

\section{Conclusion}

It is important to consider all available data in each subject presenting with an acute MI to gather as much information as possible to tailor a diagnosis and therapy focusing on the operating pathophysiological process and avoid common pitfalls. In addition, it remains of utmost importance to consider acute MI as a potential diagnosis in patients with chest pain.

Early recognition of acute $\mathrm{MI}$ is of utmost importance to lower the mortality and to reduce lasting morbidity. Insight into the potential operational mechanisms of the coronary artery incident may have a major impact on the clinical course following admission. We would like to highlight that a personalized clinical approach remains of utmost importance in each patient treated by protocolized medicine.

\section{Informed consent}

An oral informed consent was obtained from the patients to use their data in this article.

\section{Authors contribution}

FP, GJ and RA gathered data and wrote the article.

MM co-writing and editing of the article.

\section{References}

1. Egred M, Viswanathan G, Davis GK. Myocardial infarction in young adults. Postgrad Med J. 2005 Dec; 81(962): 741-5.

2. Brscic E, Bergerone S, Gagnor A, et al. Acute Myocardial Infarction in young adults. Am Heart J. 2000; 139(6): 27-31.

3. Beijk MA, Vlastra W V, Delewi R, et al. Myocardial infarction with nonobstructive coronary arteries: a focus on vasospastic angina. Neth Heart J. 2019 May; 27(5): 237-45.

4. Mannucci PM, Asselta R, Duga S, et al. The association of factor V Leiden with myocardial infarction is replicated in 1880 patients with premature disease. J Thromb Haemost. 2010; 8(10): 2116-21.

5. Lee KW, Lip GY. Acute coronary syndromes: Virchow's triad revisited. Blood Coagul Fibrinolysis. 2003 Oct; 14(7): 605-25.

6. Mostofsky E, van der Bom JG, Mukamal KJ, et al. Risk of myocardial infarction immediately after alcohol consumption. Epidemiology. 2015 Mar; 26(2): 143-50.

7. Ross GS, Bell J. Myocardial infarction associated with inappropriate use of topical cocaine as treatment for epistaxis. Am J Emerg Med. 1992 May; 10(3): 219-22.

8. Abdolrahimi SA, Sanati HR, Ansari-Ramandi MM, et al. Acute Myocardial Infarction Following Blunt Chest Trauma and Coronary Artery Dissection. Vol. 10, Journal of clinical and diagnostic research : JCDR. India; 2016. p. OD14-5.

9. Ribeiro DRP, Cambruzzi E, Schmidt MM, et al. Thrombosis in ST-elevation myocardial infarction: Insights from thrombi retrieved by aspiration thrombectomy. World J Cardiol. 2016; 8(6): 362

10. Yang XP, Reckelhoff JF. Estrogen, hormonal replacement therapy and cardiovascular disease. Curr Opin Nephrol Hypertens. 2011 Mar; 20(2): 133-8.

11. Lameijer $\mathrm{H}$, Lont MC, Buter $\mathrm{H}$, et al. Pregnancy-related myocardial infarction. Netherlands Hear J. 2017; 25(6): 365-9.

12. Jessurun GA, Tio RA, Ribbert LS, et al. Unusual cause of sudden cardiac death: basophilic degeneration of coronary arteries. Cathet Cardiovasc Diagn. 1996 0ct; 39(2): 172-6.

13. Tweet MS, Kok SN, Hayes SN. Spontaneous coronary artery dissection in women: What is known and what is yet to be understood. Clin Cardiol. 2018 Feb; 41(2): 203-10.

14. Janssen EBNJ, de Leeuw PW, Maas AHEM. Spontaneous coronary artery dissections and associated predisposing factors: a narrative review. Neth Heart J. 2019 May; 27(5): 246-51.

15. Niccoli G, Scalone G, Crea F. Acute myocardial infarction with no obstructive coronary atherosclerosis: Mechanisms and management. Eur Heart J. 2015; 36(8): 475-81. 
16. Kelbæk H. Deferred versus conventional stent implantation in patients with ST-segment elevation myocardial infarction (DANAMI 3-DEFER) an open-label, randomised controlled trial. The Lancet. 2016 May 28; 387(10034): 2199-206.
17. Van der Schoot. Acute myocardial infarction in adolescents: reappraisal of underlying mechanisms. Netherlands Heart Journal. 2020 ; 28 : 301-308 